\title{
Electron cyclotron maser emission from solar coronal funnels?
}

\author{
C. Vocks ${ }^{1,2}$ and G. Mann ${ }^{1}$ \\ 1 Astrophysikalisches Institut Potsdam, An der Sternwarte 16, 14482 Potsdam, Germany \\ 2 now at: Space Sciences Laboratory of UC Berkeley, Berkeley, CA 94720-7450, USA
}

Received 19 December 2003 / Accepted 25 February 2004

\begin{abstract}
The sun is covered by a network of supergranular cells. The convective motion of these cells leads to the formation of strong magnetic fields at the cell boundaries. At larger heights in the solar transition region and low corona, this magnetic field geometry expands rapidly within a short distance, and forms the magnetic structure of "coronal funnels". This field line geometry represents a magnetic mirror, and since the plasma density strongly increases with depth in the transition region, the electron velocity distribution function (VDF) can develop a loss cone. Within such a coronal funnel, the plasma frequency can have smaller values than the electron cyclotron frequency, $\omega_{\mathrm{p}}<\Omega_{\mathrm{e}}$. These are the necessary conditions for the generation of $\mathrm{X}$-mode waves through the electron cyclotron maser mechanism. Since there is some observational evidence for radio emission from the supergranular network, it is of interest to investigate the possibility of this plasma wave generation in a quiet stellar atmosphere in detail. In this paper, a kinetic model is used to calculate the electron VDF in a coronal funnel. A method is derived to determine wave growth rates from the electron VDF. Its application on the coronal funnel VDF indeed results in X-mode wave growth. However, it is also found that wave absorption by higher-order resonances at larger heights in the atmosphere plays an important role.
\end{abstract}

Key words. radiation mechanisms: non-thermal - Sun: corona - Sun: radio radiation - masers

\section{Introduction}

The generation of electromagnetic waves through the electron cyclotron maser mechanism is well known as a source of planetary radio emission like earth's auroral kilometric radiation (Wu \& Lee 1979) or jupiter's decametric radiation (Wu \& Freund 1977).

The electron cyclotron maser is based on the conversion of free energy provided by a loss cone distribution of the electrons into X-mode plasma waves and can be active in a plasma with a plasma frequency, $\omega_{\mathrm{p}}$, well below the electron cyclotron frequency, $\Omega_{\mathrm{e}}$ (Melrose et al. 1984). The X-mode waves are emitted nearly perpendicular to the background magnetic field (Wu \& Lee 1979; Omidi et al. 1984; Ladreiter 1991).

The electron cyclotron maser theory has not only been applied on planetary magnetospheres, but also on solar flares (Melrose \& Dulk 1982; Conway \& Willes 2000). Energetic electrons are injected near the looptops during the flare, propagate downwards and are mirrored in the magnetic field geometry that converges towards the footpoints. Electrons with low pitch angles penetrate deep into this magnetic mirror configuration. They are scattered in the cooler and denser medium of the transition region and do not return into the loop. Thus, the electron velocity distribution function (VDF) can form a loss cone in a flaring loop.

Send offprint requests to: $\mathrm{C}$. Vocks, e-mail: vocks@ssl.berkeley.edu
But it is possible that electron loss cone VDFs can be formed in the solar atmosphere also under quiet conditions without any flare activity. Coronal funnels (Gabriel 1976) are magnetic structures that are open towards the interplanetary medium. They are characterized by a rapid expansion of magnetic flux tubes in the transition region from the chromosphere towards the corona. On the one hand, in the chromosphere and below the gas pressure is much larger than the magnetic field energy density. Consequently, the convective motion of the supergranular cells accumulates the magnetic field at the borders of these cells. On the other hand, in the corona the magnetic field energy density is much larger than the gas pressure of the tenuous coronal plasma. This configuration leads to a rapid expansion of magnetic flux tubes in the transition region.

Such a magnetic field configuration acts as a magnetic mirror for coronal electrons that move sunwards. Electrons with small enough pitch angles penetrate deep into the funnel and reach the cooler and denser medium of the transition region or chromosphere. This electron population is scattered and subsequently thermalized there. It does not return into the corona, resulting in a loss cone distribution in the low corona.

In the next section it is shown that the condition $\omega_{\mathrm{p}}<\Omega_{\mathrm{e}}$ for the electron cyclotron maser can be fulfilled in a coronal funnel. Thus the question arises whether the quiet sun can be a source of radio emission through the electron cyclotron maser mechanism. There is some observational evidence for radio emission from the chromospheric network. Kosugi et al. (1986) 
report higher brightness temperatures in coronal holes at a frequency of $36 \mathrm{GHz}$ that is not found at a higher frequency of $98 \mathrm{GHz}$. Gopalswamy et al. (1999) analyze $17 \mathrm{GHz}$ observations and find a temperature enhancement of the chromosphere below coronal holes. They discuss the relation between enhanced temperatures and unipolar regions. Moran et al. (2001) observed enhanced $17 \mathrm{GHz}$ radio emission from magnetic field concentrations.

The observed frequencies are well above typical electron cyclotron frequencies in coronal funnels, e.g. $560 \mathrm{MHz}$ for a magnetic field of $B=0.02 \mathrm{~T}(200 \mathrm{G})$. But nevertheless, these observations further motivate the study whether coronal funnels are capable of emitting radio waves through the cyclotron maser mechanism even under quiet solar conditions.

We have developed a kinetic model for electrons in the solar corona and wind in order to study the interaction between whistler waves and electrons that results in an enhancement of suprathermal electron fluxes in the solar wind (Vocks \& Mann 2003). This kinetic model can also be applied on the transition region and low corona to study the loss cone formation in detail. As a kinetic model, it yields the electron VDF for each spatial location within the computation box. With these simulation results at hand it is an interesting option to investigate whether the low coronal plasma is capable of emitting X-mode waves in an efficient way.

The paper is organized as follows. In the next section, the kinetic model and its results for a coronal funnel are presented. In Sect. 3, a method to calculate the X-mode mode growth rate, $\gamma$, for a given electron VDF is derived. In Sect. 4, this method is applied on the kinetic results for a loss cone VDF in the low corona. The paper closes with the discussion and summary in Sect. 5 .

\section{A kinetic model for electrons in the solar corona}

The kinetic model we use to study the formation of electron loss cone VDFs in a coronal funnel is based on a numerical solution of the Boltzmann-Vlasov equation:

$$
\frac{\partial f}{\partial t}+(\boldsymbol{v} \cdot \nabla) f+\left(\boldsymbol{g}-\frac{e}{m_{\mathrm{e}}}(\boldsymbol{E}+\boldsymbol{v} \times \boldsymbol{B})\right) \cdot \frac{\partial f}{\partial \boldsymbol{v}}=\left(\frac{\delta f}{\delta t}\right)_{\mathrm{diff}} .
$$

$\boldsymbol{g}$ and $\boldsymbol{E}$ represent the gravitational and charge separation electric field, respectively. $\boldsymbol{B}$ is the background magnetic field, and the term on the right-hand side is a diffusion term due to Coulomb collisions and wave-particle interaction. The assumption of a gyrotropic electron VDF, $f$, simplifies the Eq. (1) considerably and reduces the phase space dimensions to $\left(v_{\|}, v_{\perp}\right)$ parallel and perpendicular to $\boldsymbol{B}$ in velocity space, and the spatial coordinate $s$ parallel to $\boldsymbol{B}$. Since the velocity coordinates are cylindrical coordinates, only positive $v_{\perp}$ need to be considered.

The kinetic model has been developed in order to study the acceleration of suprathermal electrons through resonant interaction with whistler waves in the solar corona and wind. The wave-particle interaction is described within the framework of quasilinear theory (Kennel \& Engelmann 1966). The waves enter the simulation box at the lower bound with a power law spectrum $\propto \omega^{-1}$ and propagate upwards. Inside the simulation box the spectrum evolves due to the change of the background conditions with height and due to the absorption of the waves by the electrons. At the lower bound, the spectral wave energy is chosen in such a way that it is, at very low frequencies in the MHD regime, in agreement with the spectrum of ion cyclotron waves that has been used in the coronal heating model of Vocks \& Marsch (2002).

A detailed description of the kinetic model can be found in Vocks \& Mann (2003). Here, the model is applied on the plasma of a coronal funnel that is located in the transition region and low corona.

The magnetic field geometry of the coronal funnel is adopted from the coronal funnel model of Hackenberg et al. (2000). The kinetic model includes the effects of the gravitational and the charge separation electric fields, and the diverging geometry of the coronal funnel.

The Coulomb collisions are calculated using the Landau collision integral in the form presented by Ljepojevic \& Burgess (1990). In order to yield analytic expressions for the Landau collision integral, the assumption of Maxwellian distributions of the collision partners, i.e. protons and electrons, is required. This is not a strong assumption, since the thermal cores of the distribution functions of the collision partners provide the largest contributions to the Coulomb collisions due to the $v^{-3}$ dependence of the Coulomb collision frequency on particle speed. For the same reason, the thermal cores are expected to remain close to a Maxwellian VDF even in the inhomogenous background of the transition region.

Since the kinetic model of the electrons does not yield results for the ions in the model plasma, a proton-electron fluid model is used as a background model for the kinetic calculations. The fluid model is also described in Vocks \& Mann (2003). It is based on the energy equations used in Hackenberg et al. (2000) and solves the continuity and momentum equations in a similar way like in the classical solar wind model of Parker (1958).

The fluid model also provides the charge separation electric field and enables the definition of initial and boundary conditions for the electron VDFs. They are set up as Maxwellians with densities and temperatures coincident with the fluid results. These Maxwellians are also used for calculating the Landau collision integrals for the Coulomb collisions.

In order to find a solution of the Boltzmann-Vlasov Eq. (1) that is stationary in time, the temporal evolution of the electron VDF is calculated by means of Eq. (1), starting from the Maxwellian initial condition, until a final steady state is reached.

In a self-consistent kinetic model, the moments of the electron VDF, i.e. density, drift velocity, temperature, and heat flux, would have to be considered in the fluid equations to correct the charge separation electric field and the Coulomb collision parameters. However, the computer costs for such a procedure are forbiddingly high. An accurate calculation of the moments of the electron VDF even at the lowest temperatures of the transition region as far as it is inside the simulation box would additionally require a very high resolution in the velocity coordinates of the computational mesh, thus further enhancing the computer costs. But the kinetic results and 

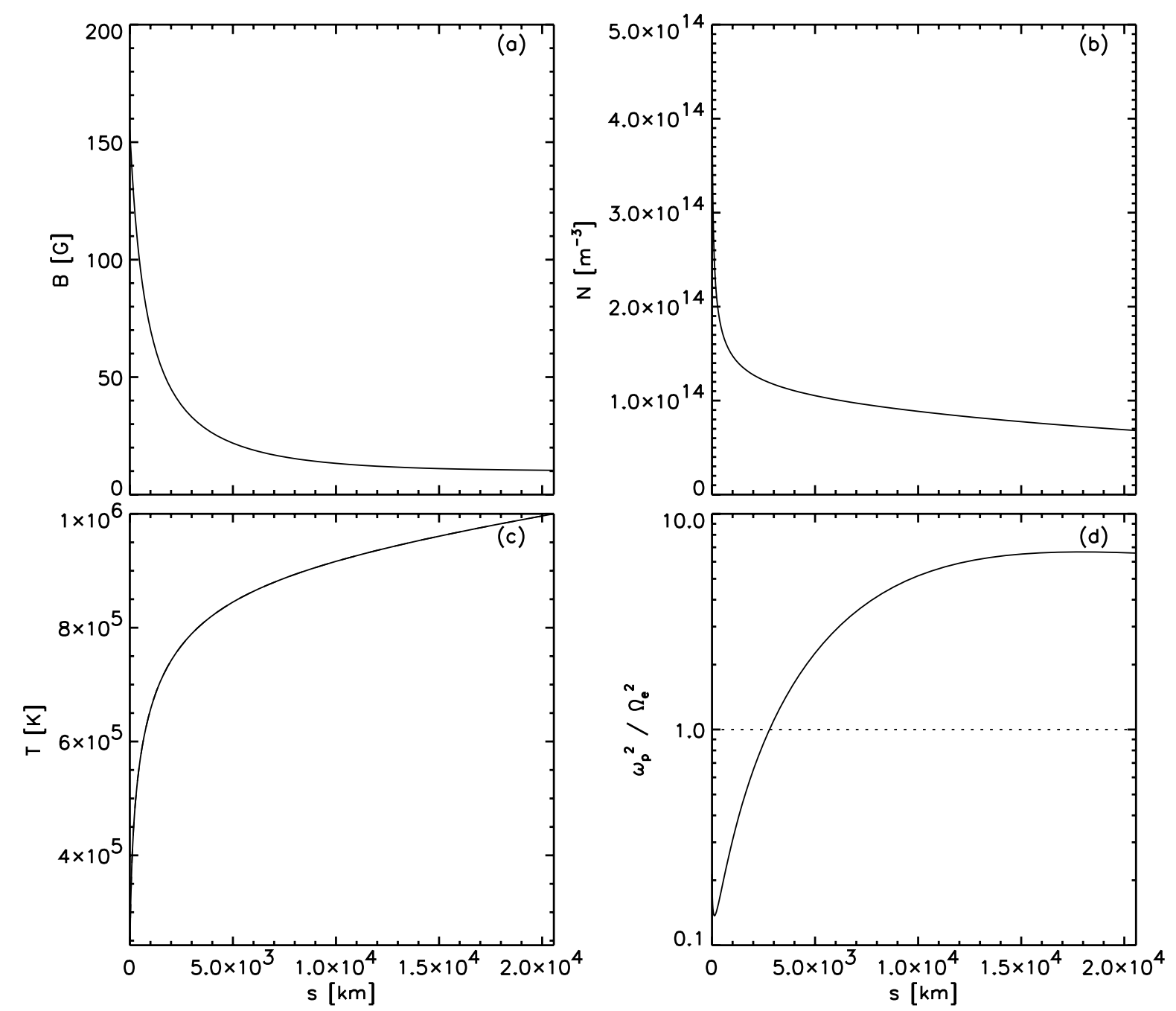

Fig. 1. Background plasma conditions for the kinetic model as functions of the height, $s$, within the simulation box. Shown are a) magnetic field, b) number density, c) temperature, and d) ratio $\omega_{\mathrm{p}}^{2} / \Omega_{\mathrm{e}}^{2}$. Protons and electrons have the same number densities and temperatures.

the background model are coupled only through the electric field and the Coulomb collision parameters. Holding the background conditions fixed does not have too much influence on the model results and just enables a numerical solution of the Boltzmann-Vlasov equation.

\subsection{The simulation box}

The lower boundary of the simulation box is located in the transition region at a temperature level of $2 \times 10^{5} \mathrm{~K}$. It extends over $20000 \mathrm{~km}$ into the low corona. Due to the assumption of a gyrotropic electron VDF, the simulation box has only one spatial coordinate $s$ along the background magnetic field, $\boldsymbol{B}$. The value $s=0$ corresponds to the lower bound of the simulation box. The velocity coordinates $v_{\|}$and $v_{\perp}$ parallel and perpendicular to $\boldsymbol{B}$, respectively, cover electron speeds up to $4.3 \times 10^{4} \mathrm{~km} \mathrm{~s}^{-1}$.

Figure 1 shows the background plasma conditions within the simulation box. The rapid expansion of the magnetic field geometry can clearly be seen in Fig. 1a as a strong decrease of the magnetic field within the lowest $5000 \mathrm{~km}$. Figures $1 \mathrm{~b}$ and 1c show the strong density and temperature gradients of the transition region.

In Fig. 1d, the square of the ratio between the plasma frequency, $\omega_{\mathrm{p}}$, and the electron cyclotron frequency, $\Omega_{\mathrm{e}}$, is plotted. The plot shows that the necessary condition for the cyclotron maser mechanism, $\omega_{\mathrm{p}}<\Omega_{\mathrm{e}}$, is fulfilled within the coronal funnel and in the lowest corona, but not at larger heights in the corona.

\subsection{Simulation results}

Within this simulation box, the electron VDF is now computed by the method based on the Boltzmann-Vlasov Eq. (1) that has been presented above. As initial condition Maxwellian electron VDFs with the same densities and temperatures as in the background conditions are defined. The temporal evolution of the electron VDFs is computed until a final steady state has been reached.

The simulation results indeed show the formation of a loss cone. It is most pronounced in the strong gradients of the transition region and fades away with height in the low corona. 


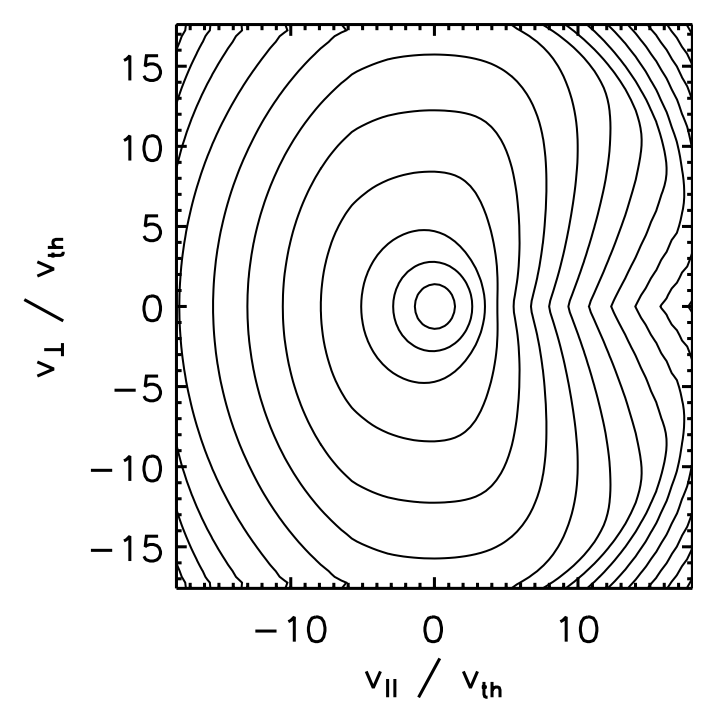

Fig. 2. Electron VDF at a height of $s=96 \mathrm{~km}$. The electron temperature at this height is $T_{\mathrm{e}}=3.88 \times 10^{5} \mathrm{~K}$, and the electron thermal speed thus $v_{\text {th }}=2420 \mathrm{~km} \mathrm{~s}^{-1}$ The isolines are chosen in such a way that they would form equidistant circles for a Maxwellian VDF.

This result is expected, since electrons that move sunwards with small pitch angles, i.e. nearly parallel to the background magnetic field, $\boldsymbol{B}$, penetrate deep into the lower transition region where the density is higher and the temperature is lower. They are scattered and thermalized there, while electrons with larger pitch angles are reflected at larger heights in the magnetic mirror of the coronal funnel. As a result, in the upper transition region and lowest portions of the corona the phase space density of electrons moving anti-sunwards with small pitch angle is reduced compared to that of electrons with larger pitch angles. Thus, a loss cone is formed. At larger heights in the corona, the Coulomb collisions fill up the loss cone, so it becomes less pronounced with height.

Figure 2 shows the electron VDF at a height of $s=96 \mathrm{~km}$ in the simulation box. This height level is located in the transition region at an electron temperature of $T_{\mathrm{e}}=3.88 \times 10^{5} \mathrm{~K}$, corresponding to an electron thermal speed of $v_{\text {th }}=2420 \mathrm{~km} \mathrm{~s}^{-1}$ Negative $v_{\perp}$ have been added to the figure by $f\left(v_{\|},-v_{\perp}\right)=$ $f\left(v_{\|}, v_{\perp}\right)$ to enhance its legibility. The loss cone is clearly visible, but it is restricted to speeds $v_{\|}>4 v_{\text {th }}$, with $v_{\text {th }}$ being the electron thermal speed. At lower speeds, a thermal core dominates.

The restriction of the loss cone to higher speeds of several thermal speeds has the consequence that only a small fraction of electron kinetic energy is available for radio wave emission. But the total number density of the electrons, $N_{\mathrm{e}}=2 \times 10^{14} \mathrm{~m}^{-3}$, is high enough to justify the assumption that the available free energy density is still capable of emitting significant radio wave power.

\section{Calculation of wave growth rates}

In the previous section it has been shown that the electron VDFs in the transition region and very low corona could be capable of emitting radio waves through the cyclotron maser mechanism. To study the efficiency of this wave generation in detail, a thorough analysis of the dispersional relation is needed. The objective of this study is to calculate wave growth rates from the plasma background conditions and the electron VDF as it is provided by the kinetic model.

\subsection{Dispersion function and wave growth rates}

The starting point for the calculation of wave growth rates is the linear theory of waves in a warm plasma as it can be found in textbooks of plasma physics, e.g. Baumjohann \& Treumann (1996). Its basics are repeated here in order to provide a comprehensive description of the theory.

The dispersion relation that links wave frequencies $\omega$ and wave vectors $\boldsymbol{k}$ for the different wave modes can be written as

$D(\omega, \boldsymbol{k})=0$

using the dispersion function $D(\omega, \boldsymbol{k})$ that is defined as:

$D(\omega, \boldsymbol{k})=\operatorname{det}\left(\frac{c^{2}}{\omega^{2}}\left(\boldsymbol{k} \boldsymbol{k}-1 k^{2}\right)+\epsilon(\omega, \boldsymbol{k})\right)$.

Note that the wave frequency, $\omega$, is complex and consists of a real part, $\omega_{\mathrm{r}}$, and the wave growth rate, $\gamma$, as imaginary part:

$\omega=\omega_{\mathrm{r}}+\mathrm{i} \gamma$.

The dispersion function is therefore also complex. A Taylor expansion around a solution $(\omega, \boldsymbol{k})$ of the dispersion relation (2) yields in the case of small $\gamma$ :

$\gamma(\omega, \boldsymbol{k})=\frac{\operatorname{Im}\left(D\left(\omega_{\mathrm{r}}, \boldsymbol{k}\right)\right)}{\left.\partial \operatorname{Re}(D(\omega, \boldsymbol{k}) / \partial \omega)\right|_{\gamma=0}}$.

Re and Im represent the real and imaginary part of a complex quantity, respectively. Equation (5) provides a method to derive the wave growth rate, $\gamma$, from the dispersion function. To calculate the dispersion function, the dielectric tensor $\epsilon(\omega, \boldsymbol{k})$ needs to be evaluated. This tensor describes the influence of the medium the wave propagates through on the wave.

In this paper, we concentrate on the $\mathrm{X}$-mode waves generated by loss cone electron VDFs. Thus, their frequencies will be in the range of typical electron frequencies like $\Omega_{\mathrm{e}}$, and resonant interaction with ions needs not to be considered here. The electrons are described by gyrotropic VDFs $f\left(v_{\|}, v_{\perp}\right)$, and the dielectric tensor can be written as:

$$
\begin{aligned}
\epsilon(\omega, \boldsymbol{k})= & \left(1-\frac{\omega_{\mathrm{p}}^{2}}{\omega^{2}}\right) 1 \\
& -\sum_{l=-\infty}^{\infty} \frac{2 \pi \omega_{\mathrm{p}}^{2}}{N_{\mathrm{e}} \omega^{2}} \int_{-\infty}^{\infty} \mathrm{d} v_{\|} \int_{0}^{\infty} v_{\perp} \mathrm{d} v_{\perp} \\
& \times\left(k_{\|} \frac{\partial f}{\partial v_{\|}}+\frac{l \Omega_{\mathrm{e}}}{v_{\perp}} \frac{\partial f}{\partial v_{\perp}}\right) \frac{\mathrm{S}_{l}\left(v_{\perp}, v_{\perp}\right)}{k_{\|} v_{\|}+l \Omega_{\mathrm{e}}-\omega}
\end{aligned}
$$

with the matrix $S_{l}$ being defined as:

$$
\mathrm{S}_{l}\left(v_{\|}, v_{\perp}\right)=\left(\begin{array}{ccc}
\frac{l^{2} \Omega_{\mathrm{e}}^{2}}{k_{\perp}^{2}} J_{l}^{2} & \mathrm{i} \frac{l v_{\perp} \Omega_{\mathrm{e}}}{k_{\perp}} J_{l} J_{l}^{\prime} & \frac{l_{\|} \Omega_{\mathrm{e}}}{k_{\perp}} J_{l}^{2} \\
-\mathrm{i} \frac{l_{\perp} \Omega_{\mathrm{e}}}{k_{\perp}} J_{l} J_{l}^{\prime} & v_{\perp}^{2}\left(J_{l}^{\prime}\right)^{2} & -\mathrm{i} v_{\|} v_{\perp} J_{l} J_{l}^{\prime} \\
\frac{l_{\|} \Omega_{\mathrm{e}}}{k_{\perp}} J_{l}^{2} & \mathrm{i} v_{\|} v_{\perp} J_{l} J_{l}^{\prime} & v_{\|}^{2} J_{l}^{2}
\end{array}\right)
$$


The index $l$ denotes the order of the resonance and $J_{l}$ represents the Bessel function of order $l, J_{l}(x)$. It is evaluated at $x=k_{\perp} v_{\perp} / \Omega_{\mathrm{e}} . J_{l}^{\prime}$ represents its derivative in $x$.

\subsection{Computation of the dielectric tensor}

The Calculation of numerical values of the dispersion function $D(\omega, \boldsymbol{k})$ requires an evaluation of the integral over electron velocity space in the definition of the dielectric tensor $\epsilon(\omega, \boldsymbol{k})$, Eq. (6). This integration is complicated by the singularity that is met when the resonance condition

$k_{\|} v_{\|}-\omega+l \Omega_{\mathrm{e}}=0$

is fulfilled. It is further important that the integrand is complex. The matrix $\mathbf{S}_{l}$ contains complex elements, but it is of a hermitian type with real eigenvalues. The more important complex quantity is the frequency, $\omega=\omega_{\mathrm{r}}+\mathrm{i} \gamma$.

Since the right hand side of Eq. (5) is evaluated only in the limit $\gamma \rightarrow 0$ we will reduce the discussion of $\epsilon(\omega, \boldsymbol{k})$ to this limit. The factors $\omega^{-2}$ in Eq. (6) then turn into $\omega_{\mathrm{r}}^{-2}$, and their imaginary parts that contain factors $\gamma$ vanish. Only the denominator $k_{\|} v_{\|}+l \Omega_{\mathrm{e}}-\omega$ with its singularity needs further attention.

To demonstrate this, the resonance frequency

$\omega_{\mathrm{res}}=k_{\|} v_{\|}+l \Omega_{\mathrm{e}}$

is introduced for brevity. In the limit $\gamma \rightarrow 0$, the real part of $\left(\omega_{\text {res }}-\omega\right)^{-1}$ simply reduces to $\left(\omega_{\text {res }}-\omega_{\mathrm{r}}\right)^{-1}$, but the imaginary part introduces the delta distribution, since

$\lim _{\gamma \rightarrow 0} \frac{\gamma}{\left(\omega_{\text {res }}-\omega_{\mathrm{r}}\right)^{2}+\gamma^{2}}=\pi \delta\left(\omega_{\text {res }}-\omega_{\mathrm{r}}\right)$.

Due to the split-up of the resonance denominator in Eq. (6) into its real and imaginary part, it is reasonable to split also the dielectric tensor $\epsilon(\omega, \boldsymbol{k})$ into a "real" and "imaginary" part:

$\epsilon=\operatorname{Re}(\epsilon)+\mathrm{i} \operatorname{Im}(\epsilon)$.

The terms "real" and "imaginary" are set in parentheses, since the matrix $S_{l}$ still introduces complex elements into both parts.

The "real" part $\operatorname{Re}(\epsilon)$ reads:

$$
\begin{aligned}
& \operatorname{Re}(\epsilon)=\left(1-\frac{\omega_{\mathrm{p}}^{2}}{\omega_{\mathrm{r}}^{2}}\right) 1-\sum_{l=-\infty}^{\infty} \frac{2 \pi \omega_{\mathrm{p}}^{2}}{N_{\mathrm{e}} \omega_{\mathrm{r}}^{2}} \\
& \quad \times \int_{-\infty}^{\infty} \mathrm{d} v_{\|} \int_{0}^{\infty} v_{\perp} \mathrm{d} v_{\perp}\left(k_{\|} \frac{\partial f}{\partial v_{\|}}+\frac{l \Omega_{\mathrm{e}}}{v_{\perp}} \frac{\partial f}{\partial v_{\perp}}\right) \frac{\mathrm{S}_{l}}{k_{\|} v_{\|}+l \Omega_{\mathrm{e}}-\omega_{\mathrm{r}}} .
\end{aligned}
$$

It still contains the singularity, Eq. (8), but this is uncritical for the integration over velocity space. The integral has a welldefined value. It is noteworthy that all electron velocities contribute to this integral. The contributions mainly depend on the values of the velocity derivatives of the electron VDF. If the VDF has no strong short-periodic (in velocity space) variations, these velocity derivatives can be expected to be highest when the $\operatorname{VDF} f\left(v_{\|}, v_{\perp}\right)$ itself has high values, i.e. within the thermal core of the VDF.

The thermal core has been found to be close to a Maxwellian VDF even in the inhomogenous background of the transition region in a coronal funnel, see Fig. 2. Thus it is reasonable to calculate $\operatorname{Re}(\epsilon)$ for a Maxwellian VDF rather than using the numerical results that suffer from a too poor resolution in velocity space in order to accurately represent the thermal core. With a Maxwellian VDF $f\left(v_{\|}, v_{\perp}\right)$, a further analytical treatment of Eq. (12) is possible that leads to the well known plasma dispersion function.

The "imaginary" part $\operatorname{Im}(\epsilon)$ of the dielectric tensor results in:

$$
\begin{aligned}
\operatorname{Im}(\epsilon)= & -\sum_{l=-\infty}^{\infty} \frac{2 \pi \omega_{\mathrm{p}}^{2}}{N_{\mathrm{e}} \omega_{\mathrm{r}}^{2}} \int_{-\infty}^{\infty} \mathrm{d} v_{\|} \int_{0}^{\infty} v_{\perp} \mathrm{d} v_{\perp} \\
& \times\left(k_{\|} \frac{\partial f}{\partial v_{\|}}+\frac{l \Omega_{\mathrm{e}}}{v_{\perp}} \frac{\partial f}{\partial v_{\perp}}\right)^{0} \mathrm{~S}_{l} \pi \delta\left(k_{\|} v_{\|}+l \Omega_{\mathrm{e}}-\omega_{\mathrm{r}}\right) .
\end{aligned}
$$

The integration over all $\left(v_{\|}, v_{\perp}\right)$ is greatly simplified by the delta distribution. It is reduced to an integral over a path in velocity space that follows the solution of the resonance condition, Eq. (8).

Since this resonance condition does not depend explicitly on $v_{\perp}$, this path seems to be simply a line over all $v_{\perp}$ at the position

$v_{\|}=\frac{\left(\omega_{\mathrm{r}}-l \Omega_{\mathrm{e}}\right)}{k_{\|}}$.

But for X-mode waves propagating nearly perpendicular to the background magnetic field, this method yields very poor results.

The reason for this is the neglect of relativistic effects that enter the resonance condition, Eq. (8), through the Lorentz factor in the electron cyclotron frequency:

$\Omega_{\mathrm{e}}=\frac{e B}{m_{\mathrm{e}}} \cdot \sqrt{1-\frac{v_{\|}^{2}+v_{\perp}^{2}}{c^{2}}}$.

Inserting this relativistic cyclotron frequency into the resonance condition, Eq. (8), and performing some algebra, finally leads to an ellipse equation in velocity space:

$\frac{\left(v_{\|}-v_{0}\right)^{2}}{a_{\|}^{2}}+\frac{v_{\perp}^{2}}{a_{\perp}^{2}}=1$

with ellipse axes

$a_{\|}=\frac{\xi c}{\sqrt{k_{\|}^{2} c^{2}+l^{2} \Omega_{0}^{2}}}, \quad a_{\perp}=\frac{\xi c}{l \Omega_{0}}$

and a center location

$v_{0}=\frac{k_{\|} \omega_{\mathrm{r}} c^{2}}{k_{\|}^{2} c^{2}+l^{2} \Omega_{0}^{2}}$

$\Omega_{0}=e B / m_{\mathrm{e}}$ is the electron cyclotron frequency in the nonrelativistic limit, and $\xi$ is defined as:

$\xi=\sqrt{l^{2} \Omega_{0}^{2}-\omega_{\mathrm{r}}^{2}+\frac{k_{\|}^{2} \omega_{\mathrm{r}}^{2} c^{2}}{k_{\|}^{2} c^{2}+l^{2} \Omega_{0}^{2}}}$.

Thus, after the relativistic correction the solutions of the resonance condition lie on an resonance ellipse in velocity space. 


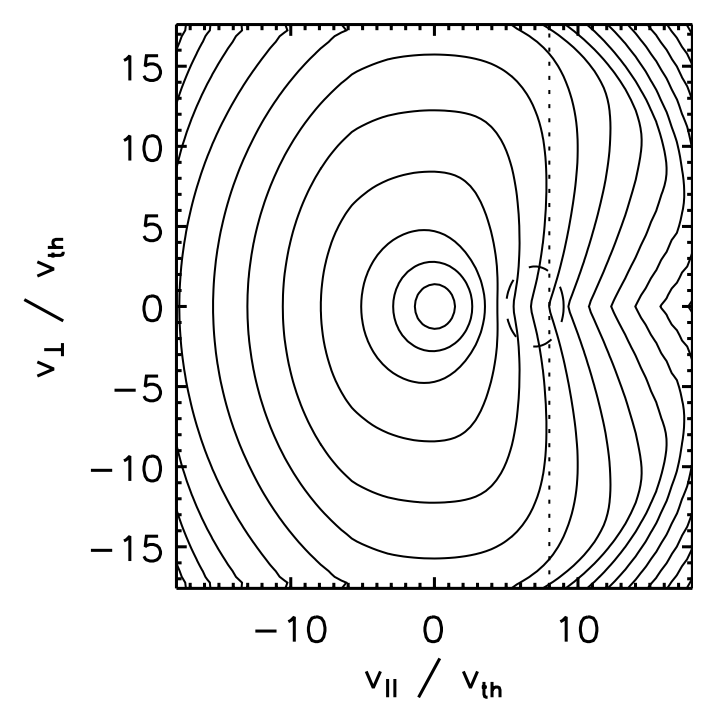

Fig. 3. Electron VDF at a height of $s=96 \mathrm{~km}$, as in Fig. 2. Shown are also a sketch of a resonance ellipse (dashed line) and an integration path for the nonrelativistic theory.

In case of the X-mode waves discussed here, the ellipse can become rather small. This is essential for the cyclotron maser mechanism (Wu \& Freund 1977) and the reason why a relativistic effect is so important for electron energies of just a few $\mathrm{keV}$ and below as they are discussed here.

Figure 3 shows the plot of the electron VDF from Fig. 2 together with a sketch of a resonance ellipse and the nonrelativistic integration path according to Eq. (14). The difference between relativistic and nonrelativistic case is considerable, in the relativistic case the whole integration path is located inside the loss cone.

For a given real part of the wave frequency, $\omega_{\mathrm{r}}$, and wavenumber $k_{\|}$, the "imaginary" part of the dielectric tensor, Eq. (13), can now be determined as a line integral along the resonance ellipse. Since this integration path does not cover the whole velocity space, local (in velocity space) features of the electron VDF, like the loss cone, can have significant influence on the "imaginary" part of the dielectric tensor for certain $\omega_{\mathrm{r}}$ and $k_{\|}$, as it is sketched in Fig. 3. Thus, in contrast to $\operatorname{Re}(\epsilon)$, the actual electron VDF as it is provided by the kinetic model has to be employed in order to determine $\operatorname{Im}(\epsilon)$.

For a given real wave frequency and wavenumber vector, $\left(\omega_{\mathrm{r}}, \boldsymbol{k}\right)$, we now have completed the calculation of the dielectric tensor $\epsilon$. Inserting this tensor into the dispersion function, Eq. (3), finally enables us to determine the wave growth rate, $\gamma$, through Eq. (5).

\section{Wave growth and absorption in the low corona}

In the previous section a method has been presented to calculate the dielectric tensor from the electron VDFs that are yielded by the kinetic model. It enables an evaluation of the dispersion function, Eq. (3), and thus with Eq. (5) of the wave growth rates, $\gamma$.

In this section this method is applied on the transition region electron VDF that was obtained with the kinetic model. The wave growth rates, $\gamma$, are calculated as functions of the

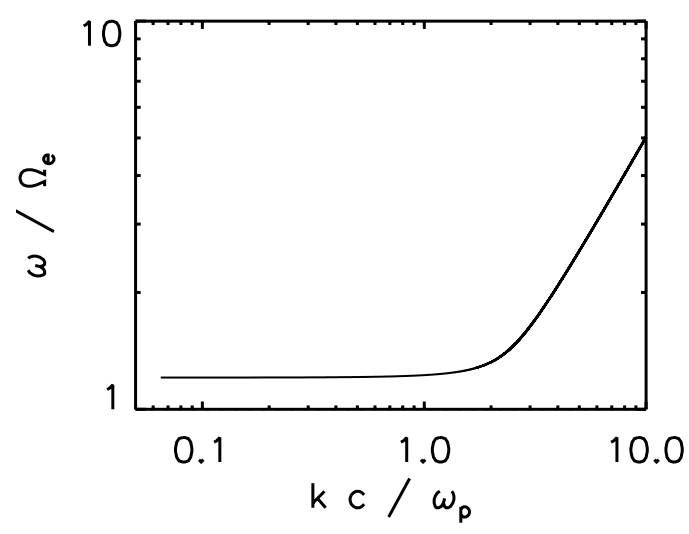

Fig. 4. Dispersion relation of the X-mode propagating perpendicular to the background magnetic field in a plasma with $\omega_{\mathrm{p}}^{2} / \Omega_{\mathrm{e}}^{2}=0.25$.

wave frequency $\omega$ and the angle $\theta$ between the wave vector and the background magnetic field. This requires the determination of a wave number, $k$, for each $(\omega, \theta)$ by the dispersion relation, Eq. (2). We consider only waves propagating away from the sun, i.e. with $k_{\|} \geq 0$, that possibly could be observed in interplanetary space or on earth.

Figure 4 shows the dispersion relation of $\mathrm{X}$-mode waves propagating perpendicular to the background magnetic field. The ratio between plasma frequency and electron cyclotron frequency has been chosen as $\omega_{\mathrm{p}}^{2} / \Omega_{\mathrm{e}}^{2}=0.25$, which is a typical value for the coronal funnel, see Fig. 1d.

The necessary condition for cyclotron maser emission, $\omega_{\mathrm{p}}^{2}<\Omega_{\mathrm{e}}^{2}$, is fulfilled, but not $\omega_{\mathrm{p}}^{2} \ll \Omega_{\mathrm{e}}^{2}$ (Ladreiter 1991). This has an important influence on the dispersion relation. The X-mode branch does not start at $\omega=\Omega_{\mathrm{e}}$, but well above $\Omega_{\mathrm{e}}$. Since the wave emission at the resonance of the order $l$ is restricted to a small region around $l \Omega_{\mathrm{e}}$ by the resonance condition, Eq. (8), no wave generation on the fundamental mode, $l=1$, is possible in the coronal funnel.

\subsection{Wave emission at $\omega \approx 2 \Omega_{e}$}

For this reason, wave emission is only possible at the order $l=2$ or higher. So we consider the case $l=2$ and calculate the wave growth rate, $\gamma$, for the electron VDF in the coronal funnel that is shown in Fig. 2.

Figure 5 displays the wave growth rates that result from this electron VDF. $\gamma$ has positive values in a small region in frequency space. This region extends over all propagation angles $\theta$, and the maximum values of $\gamma$ increase with $\theta$. Thus waves propagating nearly perpendicular to the background magnetic field are generated preferredly, as it is expected for the cyclotron maser mechanism. But as $\theta \rightarrow 90^{\circ}$, the frequency range of the wave generation region becomes very small and falls below the differences in resonance frequency that correspond to adjacent points in velocity space of the computational grid of the kinetic model for the electrons.

This plot shows that the electron cyclotron maser mechanism is active and can produce $\mathrm{X}$-mode waves in a coronal funnel under quiet solar conditions. 


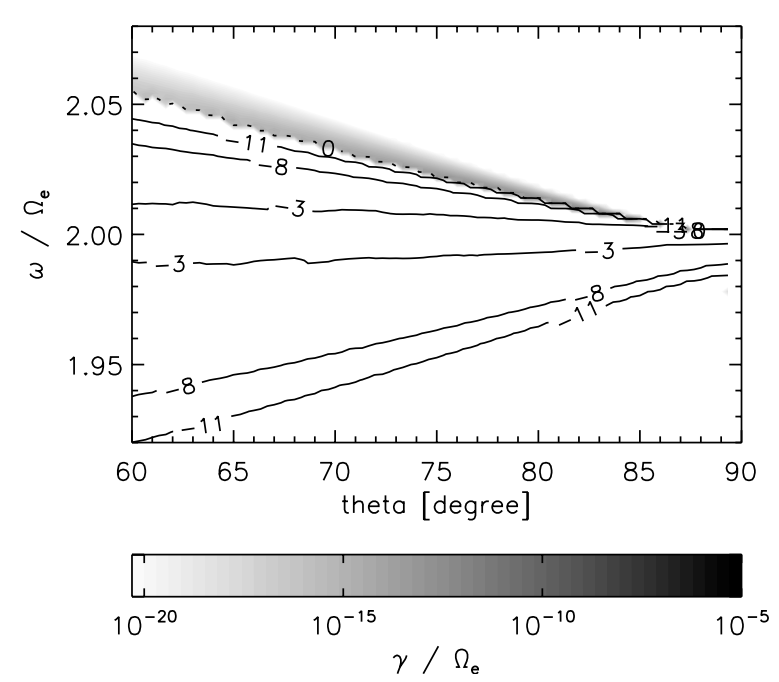

Fig. 5. Growth rates of X-mode waves in the coronal funnel at $s=$ $96 \mathrm{~km}$ as function of wave frequency, $\omega$, and propagation angle, $\theta$. Positive growth rates, $\gamma>0$, are displayed as a greyscale plot, and negative growth rates, $\gamma<0$, by isolines marked with numbers that denote an exponent, e.g. -3 corresponds to $\gamma / \Omega_{\mathrm{e}}=-10^{-3}$.

The split of the frequency space into a region with wave growth at high frequencies and into a region with wave absorption around and below the twofold electron cyclotron frequency is a consequence of the resonance condition, Eq. (8), with the relativistic correction, Eq. (15). The resonance frequency, $\omega_{\text {res }}$, is determined by:

$\omega_{\text {res }}=l \Omega_{\mathrm{e}}+k_{\|} v_{\|}$.

The higher $v_{\|}$, the higher is $\omega_{\text {res }}$. The plot of the electron VDF, Fig. 2, shows that the loss cone and thus the region in velocity space that provides free energy to the electron cyclotron maser mechanism, is restricted to high positive $v_{\|}$. This is the reason for the wave growth at high frequencies. This wave growth region is restricted towards even higher frequencies by the decrease of the phase space density of the electron VDF at the correspondingly high $v_{\|}$.

Waves with lower frequencies $\omega \approx 2 \Omega_{\mathrm{e}}$ interact with electrons with lower $v_{\|}$, where the VDF is close to a Maxwellian or Bi-Maxwellian. This portion of the VDF provides no energy for wave growth. Moreover, such waves suffer relatively strong damping with $\gamma>10^{-3} \Omega_{\mathrm{e}}$, as can be seen in Fig. 5 .

This wave damping at frequencies $\omega \approx 2 \Omega_{\mathrm{e}}$ and below does not prevent the waves generated at higher frequencies from escaping into interplanetary space. In a coronal funnel and in the corona, the magnetic field decreases with height and thus along the path of wave propagation. So the frequency of a wave that is generated by the cyclotron maser mechanism, and propagates away from the sun, increases in units of the local electron cyclotron frequency. This has the consequence that the wave leaves the region of positive $\gamma$ in Fig. 5 towards a frequency domain where no wave-particle interaction and thus no absorption takes place.

However, the positive $\gamma$ in Fig. 5 are very small, with a maximum of $10^{-8} \Omega_{\mathrm{e}}$. This is due to the restriction of the loss cone in Fig. 2 to higher electron speeds of several $v_{\text {th }}$. At the

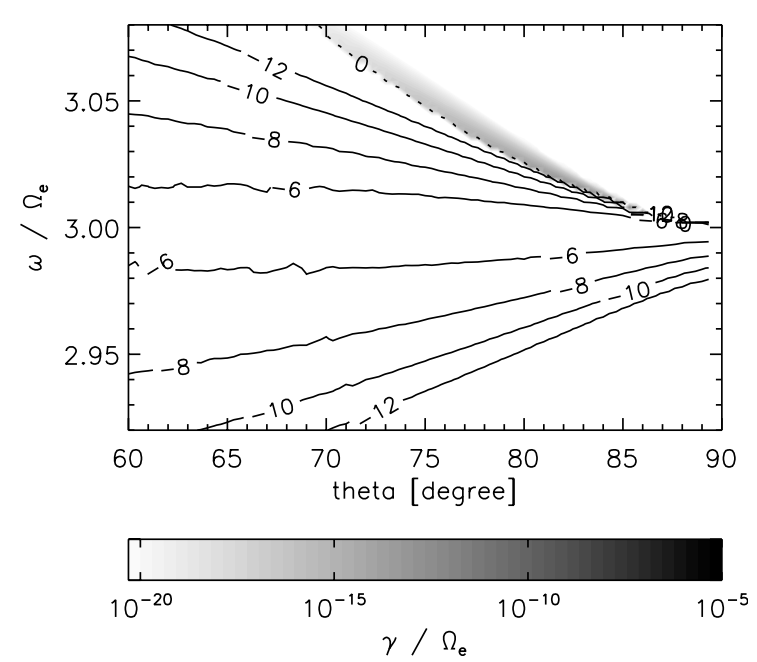

Fig. 6. Growth rates of X-mode waves in the coronal funnel at $s=$ $513 \mathrm{~km}$ as function of wave frequency, $\omega$, and propagation angle, $\theta$. Positive growth rates, $\gamma>0$, are displayed as a greyscale plot, and negative growth rates, $\gamma<0$, by isolines marked with numbers that denote an exponent, e.g. -6 corresponds to $\gamma / \Omega_{\mathrm{e}}=-10^{-6}$.

speeds where the loss cone appears, the phase space density of the electrons is already small compared to the thermal bulk. This results in only a few electrons contributing to the wave growth. So only weak wave emission can be expected from this electron cyclotron maser.

\subsection{Wave emission and absorption at $\omega \approx 3 \Omega_{e}$}

As a wave that has been generated by the $l=2$ resonance propagates further up into the corona, its frequency $\omega$ remains constant, but the magnetic field and thus $\Omega_{\mathrm{e}}$ continues to decrease. At a certain height, the condition $\omega=3 \Omega_{\mathrm{e}}$ is fulfilled. For waves emitted at $s=96 \mathrm{~km}$, this is the case at a height $s=513 \mathrm{~km}$.

Now the wave interacts with the electrons through the $l=3$ resonance. Since the magnetic field and $\Omega_{\mathrm{e}}$ continuously vary with height, the wave successively passes layers where its frequency is slightly below, equal to, and slightly above $3 \Omega_{\mathrm{e}}$.

Figure 6 shows the wave growth rates for $l=3$ at a height of $s=513 \mathrm{~km}$. At a first glance, the picture looks similar to Fig. 5 for $l=2$ and $s=96 \mathrm{~km}$. At higher frequencies waves are generated, since the loss cone in the electron VDF is still present. But due to the lower density at $s=513 \mathrm{~km}$ and the lower efficiency of the wave-particle interaction at $l=3$ both the wave absorption rates as well as the wave growth rates are smaller here.

Nevertheless, the wave absorption rate at $\omega=3 \Omega_{\mathrm{e}}$ is still some orders of magnitude higher than the maximum wave growth rate in the $l=2$ case. The wave absorption at $l=3$ is much stronger than the emission at $l=2$. It follows that a wave that is emitted in the coronal funnel by the $l=2$ resonance can be damped at $l=3$ down to a level below the initial background fluctuation that started growing at $l=2$ through the cyclotron maser mechanism.

Figure 6 shows some weak wave emission at frequencies little above $3 \Omega_{\mathrm{e}}$, but these waves also will interact with the 
next higher, i.e. $l=4$, resonance at some larger height. There, the wave absorption again will exceed the emission at $l=3$, so that these waves also cannot escape into interplanetary space. If the electron VDF still has the loss cone at the $l=4$ level, some wave emission might be possible, but these waves are absorbed at $l=5$, and so forth. The loss cone disappears with height above the coronal funnel, and the plasma frequency, $\omega_{\mathrm{p}}$, eventually becomes larger than $\Omega_{\mathrm{e}}$ at a certain level, see Fig. 1. Since the necessary condition $\omega_{\mathrm{p}}<\Omega_{\mathrm{e}}$ is not fulfilled, no wave generation is possible above this level.

To summarize, these model calculations show that X-mode wave generation through the cyclotron maser mechanism is possible in a coronal funnel. But higher-order resonances absorb these waves at larger heights in the corona, so they cannot escape into interplanetary space.

\section{Discussion and summary}

The rapidly opening magnetic structure of a coronal funnel provides the geometry of a magnetic mirror, and the condition $\omega_{\mathrm{p}}<\Omega_{\mathrm{e}}$ is found to be fulfilled in the transition region and low corona within this funnel. So it is reasonable to suppose and worth to investigate whether the quiet solar atmosphere is capable of generating radio waves through the electron cyclotron maser mechanism in coronal funnels. The electron cyclotron maser mechanism is well known to be active in other space plasmas, as it generates jupiter's decametric radiation (Wu \& Freund 1977) or earth's auroral kilometric radiation (Wu \& Lee 1979).

The electron VDF in a coronal funnel is calculated by a kinetic model that solves the Boltzmann-Vlasov equation for the electrons. The VDF shows the expected loss cone. The condition $\omega_{\mathrm{p}}<\Omega_{\mathrm{e}}$ is also fulfilled at the same height within the funnel, so the cyclotron maser mechanism can be active. The question is: is it efficient enough to produce significant wave power that could be observed?

To answer this question, a method to derive the wave growth rate $\gamma$ from the electron VDF is developed. The application of this method on the coronal funnel plasma shows that the electron cyclotron maser mechanism is indeed active at the resonance orders $l=2$ and higher, but the wave growth rates are small. The waves can escape from the site of their generation, but they are absorbed at a height level where they interact with the plasma through the resonance of next higher order. Thus an emission of X-mode waves from coronal funnels at the borders of the supergranular network seems not to be possible.

The reason for the low wave growth rates that are smaller than the absorption rates at the next higher resonance is the low phase space density of that portion of the electron VDF that shows the loss cone. The loss cone is restricted to higher speeds $v_{\|}$as can be seen in Fig. 2, so its phase space density is much smaller than that of the thermal bulk, that is mainly responsible for the wave absorption at the next higher resonance.
Of course the exact shape of the electron VDF depends on the coronal funnel model assumptions and parameters. However, due to the strong dependence of the Coulomb collision frequency on the electron velocity that scales with $v^{-3}$, it cannot be expected that it is possible to extend the loss cone significantly towards the thermal core, and thus to enhance the wave growth rates by modifying the funnel geometry.

In a solar flare the conditions may be different and enable more efficient wave generation through the injection of energetic electrons from the tops of the flaring loop, as it is described by Melrose \& Dulk (1982). Thus the sun can produce radio waves through the cyclotron maser mechanism. However, under quiet solar conditions we do not find the fulfillment of such conditions as $\omega_{\mathrm{p}} \ll \Omega_{\mathrm{e}}$ for wave generation on the fundamental mode, or a loss cone in a region of the electron VDF with a sufficiently high phase space density.

So it has to be concluded that the electron cyclotron maser mechanism is not able to provide significant radio wave emission from the coronal funnels at the supergranular network, despite the fulfillment of the necessary conditions for the maser in that portion of the solar atmosphere.

Acknowledgements. This work was financially supported by the Deutsches Zentrum für Luft- und Raumfahrt (DLR) under grant 50 OC 9702.

\section{References}

Baumjohann, W., \& Treumann, R. A. 1996, Basic Space Plasma Physics (London: Imperial College Press)

Conway, A. J., \& Willes, A. J. 2000, A\&A, 355, 751

Gabriel, A. H. 1976, Phil. Trans. R. Soc. London, Ser. A, 281, 339

Gopalswamy, N., Shibasaki, K., Thompson, B. J., Gurman, J. B, Deforest, C. E. 1999, Is the Chromosphere Hotter in Coronal Holes? In Proceedings of the Ninth International Solar Wind Conference, AIP Conf. Proc., 277

Hackenberg, P., Marsch, E., \& Mann, G. 2000, A\&A, 360, 1139

Kennel, C. F., \& Engelmann, F. 1966, Phys. Fluids, 9, 2377

Kosugi, T., Ishiguro, M., \& Shibasaki, K. 1986, Publ. Astron. Soc. Japan, 38, 1

Ladreiter, H. P. 1991, ApJ, 370, 419

Ljepojevic, N. N., \& Burgess, A. 1990, Proc. R. Soc. Lond. A, 428, 71

Melrose, D. B., \& Dulk, G. A. 1982, ApJ, 259, 844

Melrose, D. B., Hewitt, R. G., \& Dulk, G. A. 1984, J. Geophys. Res., 89, 897

Moran, T., Gopalswamy, N., Dammasch, I. E., \& Wilhelm, K. 2001, A\&A, 378, 1037

Omidi, N., Gurnett, D. A., \& Wu, C. S. 1984, J. Geophys. Res., 89, 883

Parker, E. N. 1958, ApJ, 128, 664

Vocks, C., \& Marsch, E. 2002, ApJ, 568, 1030

Vocks, C., \& Mann, G. 2003, ApJ, 593, 1134

Wu, C. S., \& Freund, H. P. 1977, ApJ, 213, 575

Wu, C. S., \& Lee, L. C. 1979, ApJ, 230, 621 